\title{
Distributed Predictive Control of the 7-Machine CIGRÉ Power System
}

\author{
R. M. Hermans, Student Member, IEEE
}

\author{
M. Lazar, Member, IEEE
}

\begin{abstract}
Stable operation of the future electrical power system will require efficient techniques for supply-demand balancing, i.e., load-frequency control, due to liberalization of electrical energy production. Currently, there is a growing interest for asymptotically stabilizing the grid frequency via model predictive control (MPC). However, the centralized implementation of standard MPC is hampered by the scale and complexity of power networks. In this paper we therefore evaluate the suitability of a scalable, distributed Lyapunovbased MPC algorithm as an alternative to conventional balancing techniques. The approach is particularly suited for largescale power networks, as it employs only local information and limited communication between directly-coupled generator buses to provide a stabilizing control action. The effectiveness of the distributed control scheme is assessed by simulating it in closed-loop with the 7-machine CIGRÉ benchmark system.
\end{abstract}

\section{INTRODUCTION}

The growing need for a more efficient, expanding electricity infrastructure has resulted in a fundamental restructuring of the power grid. Firstly, from a regulated, monopolistic operation, power systems are being deregulated and reorganized to support large numbers of market actors that compete for energy supply and demand, which is believed to lower prices and minimize costs (see, e.g., [1]). Secondly, the past decennium has shown a considerable increase of distributed generation (DG) at the expense of conventional, large-scale power plants. The penetration of (often renewable-based) DG is expected to continue as a result of environmental concerns and rising fossil fuel prices, see, e.g., [2].

The major shift in electricity grid design requires a fundamental change of control strategies, in order to guarantee reliable energy supply in the future. One of the control problems complicated by deregulation and DG is that of $a u-$ tomatic generation control (AGC), see, e.g., [3]. AGC aims at asymptotic stabilization of the network frequency, which corresponds to continuously balancing generation and load. Today, AGC is usually implemented via classical proportionalintegral feedback laws. This approach has proven to be reasonably adequate for traditional power systems that are characterized by highly repetitive power flows, a relatively small amount of uncertain fluctuations on the demand side and well-controllable, large-scale power plants on the supply side. However, with grid operation becoming deregulated, competitive economic forces tend to push the system towards its stability boundaries (see, e.g., [1]). Recent studies, such as [4], [5], show that already today, the increasing overlap

R. M. Hermans, M. Lazar and A. Jokić are with the Department of Electrical Engineering, Eindhoven University of Technology, P.O. Box 513, 5600 MB Eindhoven, The Netherlands, E-mails: $\{r . m . h e r m a n s$, m.lazar, a.jokic\}@tue.nl. of market and control mechanisms is inducing alarmingly high frequency fluctuations in the European electricity grid, thereby impounding a large part of the balancing reserves. In addition, the performance of conventional AGC is threatened considerably by the increasing extent of unpredictable imbalance fluctuations that originate from intermittent DG.

Recently, it was observed that model predictive control (MPC) has a potential for solving the problems that are expected in future electrical power networks, see [6]-[8]. MPC can explicitly take state/input constraints into account and can employ disturbance models to counteract imbalance fluctuations due to intermittent distributed generation or market-based scheduling. Yet, the fact that MPC is a centralized control method is a major issue if it is to be used in power systems. Standard MPC requires a single controller to measure all the system outputs and to compute and apply the control action to all actuators in the network, all within one sampling period. As power networks are large and complex, it is practically impossible to implement a dedicated centralized predictive control scheme. This motivates the search for non-centralized formulations of MPC, in which the overall control action is formed by a set of control laws, each assigned to a separate control area.

The non-centralized MPC schemes that have been proposed in the literature can roughly be divided into decentralized techniques, see, e.g., [9]-[11], in which local controllers operate without communication (just as standard AGC), and distributed techniques, see, e.g., [6], [7], [12], [13], in which the control action is computed by exploiting a mutual exchange of information over a usually predefined structured communication network. Distributed methods that employ iterations or global information, such as the approaches proposed in [6], [7], generally outperform decentralized MPC in terms of optimality with respect to a global objective at the cost of higher computational and communication requirements. However, the sampling periods required in power system control (in the order of seconds) are usually too short for MPC algorithms to perform iterations or to exchange global information in a reliable fashion, see [14]. Consequently, the objective of a globally optimal performing MPC-controlled power system is currently out of reach.

Given these observations, we focus on load-frequency control using the non-iterative distributed Lyapunov-based MPC scheme that was presented in [15]. This method needs no global coordination to guarantee stability and can be implemented in an almost-decentralized fashion. That is, the controller only requires one run of information exchange between directly neighboring subsystems per sampling instant. This communication scheme fits the current power 
system control architecture, as present transmission lines are usually equipped with communication links. We illustrate the effectiveness of the almost-decentralized scheme of [15] by simulating it in closed-loop with the 7-machine CIGRÉ system that is often used in load-frequency control studies.

\section{A. Basic Notions and Definitions}

Let $\mathbb{R}, \mathbb{R}_{+}, \mathbb{Z}$ and $\mathbb{Z}_{+}$be the sets of real, non-negative real, integer and non-negative integer numbers, respectively. For every $c \in \mathbb{R}$ and $\Pi \subseteq \mathbb{R}$ we define $\Pi_{\geq c}:=\{k \in \Pi \mid k \geq c\}$ and $\Pi_{\leq c}:=\{k \in \Pi \mid k \leq c\}$. Moreover, let $\mathbb{Z}_{\Pi}:=\{k \in \mathbb{Z} \mid$

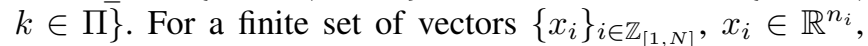
$N \in \mathbb{Z}_{\geq 1}$, let $\operatorname{col}\left(\left\{x_{i}\right\}_{\left.i \in \mathbb{Z}_{[1, N]}\right)}\right.$ or $\operatorname{col}\left(x_{1}, \ldots, x_{N}\right)$ be the column vector $\left(x_{1}^{\top}, \ldots, x_{n}^{\top}\right)^{\top}$, and let $\operatorname{diag}\left(x_{i}\right)$ denote a square matrix with the elements of $x_{i}$ on the main diagonal and zeros elsewhere. Let $\mathbf{0}_{n}$ and $\mathbf{1}_{n}$ denote column vectors in $\mathbb{R}^{n}$ with all elements equal to zero and one, respectively. $I_{N} \in \mathbb{R}^{N \times N}$ is the identity matrix. For a set $\mathcal{S} \subseteq \mathbb{R}^{n}$, the interior of $\mathcal{S}$ is represented by $\operatorname{int}(\mathcal{S})$. For $x \in \mathbb{R}^{n}$, let $\|x\|$ denote an arbitrary $p$-norm and let $[x]_{i}, i \in \mathbb{Z}_{[1, n]}$ be the $i$-th component of $x$. The $\infty$-norm of $x$ is $\|x\|_{\infty}:=$ $\max _{i=1, \ldots, n}\left|[x]_{i}\right|$, where $|\cdot|$ is the absolute value operator. For $M \in \mathbb{R}^{m \times n}$, let $\|M\|:=\max _{x \neq \mathbf{0}_{n}} \frac{\|M x\|}{\|x\|}$ be the induced matrix norm. If $m=n, M \succ 0$ and $M \succeq 0$ indicates that $M$ is positive definite and positive semi-definite, respectively. A function $\varphi: \mathbb{R}_{+} \rightarrow \mathbb{R}_{+}$belongs to class $\mathcal{K}$ if it is continuous, strictly increasing and $\varphi(0)=0$. Function $\varphi \in \mathcal{K}$ is in class $\mathcal{K}_{\infty}$ if $\lim _{s \rightarrow \infty} \varphi(s)=\infty$. A set-valued map on $\mathcal{S}_{1} \subseteq \mathbb{R}^{n}$ to $\mathcal{S}_{2} \subseteq \mathbb{R}^{m}$ is denoted by $\mathcal{S}_{1} \rightrightarrows \mathcal{S}_{2}$.

\section{Multimachine Power Systems}

$N$-machine power systems, such as the network shown in Fig. 1, consist of $N$ generator buses and a finite number of load buses that are interconnected by a grid of transmission lines. These networks can be described by a connected graph $\overline{\mathcal{G}}=(\overline{\mathcal{S}}, \overline{\mathcal{E}}, A)$, with a collection of buses/nodes $\overline{\mathcal{S}}:=$ $\left\{\mathcal{S}_{\text {Generator }}, \mathcal{S}_{\text {Load }}\right\}=\left\{\left\{\varsigma_{1}, \ldots, \varsigma_{N}\right\},\left\{\varsigma_{N+1}, \ldots, \varsigma_{M}\right\}\right\}$, a set of tie lines/undirected edges $\overline{\mathcal{E}} \subseteq\left\{\left(\varsigma_{i}, \varsigma_{j}\right) \in \overline{\mathcal{S}} \times \overline{\mathcal{S}} \mid i \neq j\right\}$ and a weighted adjacency matrix $A \in \mathbb{R}^{M \times M}$. Edges are denoted by $\varepsilon_{i j}:=\left(\varsigma_{i}, \varsigma_{j}\right)$ and $A$ satisfies $[A]_{i j} \neq 0 \Leftrightarrow \varepsilon_{i j} \in$ $\overline{\mathcal{E}}$. For convenience, we define the weights in the adjacency matrix as $[A]_{i j}:=-b_{i j}$, where $b_{i j}\left[\Omega^{-1}\right]$ is the susceptance (i.e., the inverse inductive reactance) of line/edges $\varepsilon_{i j}, \varepsilon_{j i}$.

The linearized continuous-time dynamics of steam-valve controlled generators can be described by the following standard model for load-frequency control studies, see [3]:

$$
\begin{aligned}
\dot{\delta}_{i} & =\omega_{i} \\
\dot{\omega}_{i} & =\frac{1}{H_{i}}\left(P_{\mathrm{M}_{i}}-D_{i} \omega_{i}-P_{\mathrm{L}_{i}}-\sum_{\left\{j \mid\left(\varsigma_{i}, \varsigma_{j}\right) \in \overline{\mathcal{E}}\right\}} P_{\mathrm{tie}_{i j}}\right), \\
\dot{P}_{\mathrm{M}_{i}} & =\frac{1}{\tau_{\mathrm{T}_{i}}}\left(P_{\mathrm{G}_{i}}-P_{\mathrm{M}_{i}}\right) \\
\dot{P}_{\mathrm{G}_{i}} & =\frac{1}{\tau_{G_{i}}}\left(P_{\mathrm{ref}_{i}}-P_{\mathrm{G}_{i}}-\frac{1}{r_{i}} \omega_{i}\right) .
\end{aligned}
$$

Here, $\delta_{i}[\mathrm{rad}], \omega_{i}[\mathrm{rad} / \mathrm{s}], P_{\mathrm{M}_{i}}[\mathrm{MW}]$ and $P_{\mathrm{G}_{i}}[\mathrm{MW}]$ are the rotor/voltage phase angle and frequency, and the turbine and governor states of the machine (or lumped set of machines) at bus $\varsigma_{i} \in \mathcal{S}_{\text {Generator }}$, respectively, all measured with respect to the a priori set values $\delta_{i, 0}, \omega_{0}, P_{\mathrm{M}_{i, 0}}$ and $P_{\mathrm{G}_{i, 0}}$. The relative control input of system $i$ is $P_{\text {ref }_{i}}[\mathrm{MW}]$ and the exogenous disturbance input $P_{\mathrm{L}_{i}}$ [MW] is the deviation in power demand with respect to the operating point. The parameters of generator bus $i$ are $H_{i}, D_{i}, \tau_{\mathrm{T}_{i}}, \tau_{G_{i}}$ and $r_{i}$, i.e., the inertia, damping coefficient, turbine and governor time constants, and the regulation constant of the primary, decentralized feedback loop, respectively. The power flow from bus $\varsigma_{i}$ to connected nodes $\left\{\varsigma_{j} \in \overline{\mathcal{S}} \mid\left(\varsigma_{i}, \varsigma_{j}\right) \in \overline{\mathcal{E}}\right\}$ is determined using a "DC power flow" model, see [16], which is an acceptable approximation of the realistic nonlinear "AC power flow" equations if small phase differences are considered. Hence, the flow in line $\varepsilon_{i j} \in \overline{\mathcal{E}}$ is given by $P_{\text {tie }_{i j}}=b_{i j}\left(\delta_{i}-\delta_{j}\right)=-P_{\mathrm{tie}_{j i}}$, where $P_{\mathrm{tie}_{i j}}>0$ indicates power flow from $i$ to $j$.

At frequency-control relevant time scales, load-bus angle dynamics can be ignored, as the inertia at these nodes is negligibly small compared to that of generators. Thus, the coupling between $\delta_{j}, j \in\left\{j \mid \varsigma_{j} \in \mathcal{S}_{\text {Load }}\right\}$, and $\delta_{i}, \omega_{i}, i \in$ $\left\{i \mid \varsigma_{i} \in \mathcal{S}_{\text {Generator }}\right\}$ is approximated by

$$
\left[\begin{array}{c}
H_{1} \dot{\omega}_{1} \\
\vdots \\
\frac{H_{N} \dot{\omega}_{N}}{0} \\
\vdots \\
0
\end{array}\right]=\left[\begin{array}{c}
P_{\mathrm{M}_{1}}-D_{1} \omega_{1}-P_{\mathrm{L}_{1}} \\
\vdots \\
\frac{P_{\mathrm{M}_{N}}-D_{N} \omega_{N}-P_{\mathrm{L}_{N}}}{-P_{\mathrm{L}_{N+1}}} \\
\vdots \\
-P_{\mathrm{L}_{M}}
\end{array}\right]-\left[\begin{array}{l|l}
B_{11} & B_{12} \\
B_{21} & B_{22}
\end{array}\right]\left[\begin{array}{c}
\delta_{1} \\
\vdots \\
\frac{\delta_{N}}{\delta_{N+1}} \\
\vdots \\
\delta_{M}
\end{array}\right],
$$

where $B=\left[\begin{array}{ll}B_{11} & B_{12} \\ B_{21} & B_{22}\end{array}\right]:=A-\operatorname{diag}\left(A \mathbf{1}_{M}\right) \in \mathbb{R}^{M \times M}$. Eliminating $\delta_{N+1}, \ldots, \delta_{M}$ from (2) reduces (1b) to

$$
\left[\begin{array}{c}
H_{1} \dot{\omega}_{1} \\
\vdots \\
H_{N} \dot{\omega}_{N}
\end{array}\right]=\left[\begin{array}{c}
P_{\mathrm{M}_{1}}-D_{1} \omega_{1} \\
\vdots \\
P_{\mathrm{M}_{N}}-D_{N} \omega_{N}
\end{array}\right]-\Gamma\left[\begin{array}{c}
\delta_{1} \\
\vdots \\
\delta_{N}
\end{array}\right]+\Upsilon\left[\begin{array}{c}
P_{\mathrm{L}_{1}} \\
\vdots \\
P_{\mathrm{L}_{M}}
\end{array}\right],
$$

where $\Gamma:=\left(B_{11}-B_{12} B_{22}^{-1} B_{21}\right) \in \mathbb{R}^{N \times N}$ and $\Upsilon:=$ $\left[\begin{array}{ll}-I_{N} & B_{12} B_{22}^{-1}\end{array}\right]$. Hence, given the sets $\mathcal{S}:=\mathcal{S}_{\text {Generator }}$ and $\mathcal{E}:=\left\{\left(\varsigma_{i}, \varsigma_{j}\right) \in \mathcal{S} \times \mathcal{S} \mid i \neq j,[\Gamma]_{i j} \neq 0\right\}$, the power system can be defined as a network of dynamical systems (NDS) with connected graph representation $\mathcal{G}=(\mathcal{S}, \mathcal{E})$, where the continuous-time dynamics of the systems assigned to vertices $\varsigma_{i} \in \mathcal{S}$ are given by (1a), (1c)-(1d) and (3).

Now consider the following control problem.

Problem II.1 Single-area load-frequency or automaticgeneration control $(A G C)$ : Find a control law for a singlearea multimachine power system that asymptotically stabilizes the network bus frequency at nominal value 0 .

Load-frequency control is crucial for stable power system operation, particularly since asymptotic frequency stabilization corresponds to continuously balancing active power and energy supply/demand, see, e.g., [3]. This is necessary as efficient, economically feasible ways of storing electrical energy are scarce. Moreover, to ensure safe operation of transformers and synchronous machines in the network, voltage frequency fluctuations should be small. Also, AGC allows to regulate the inter-area power flow, which is important because the corresponding transfer capacity is limited.

Because conventional AGC performance is affected by deregulation, network growth and the introduction of DG, 
we suggest to solve Prob. II.1 with the non-centralized MPC scheme that was recently proposed in [15] instead.

\section{Almost-Decentralized Predictive Control}

The control synthesis method employed in this work is Lyapunov-based MPC, see [17], which is a successful class of predictive control techniques when stabilization is the main focus. The interested reader is referred to the appendix for a short overview on the underlying notions of Lyapunov stability and control Lyapunov functions (CLFs).

Consider a NDS described by the graph $\mathcal{G}=(\mathcal{S}, \mathcal{E})$, where the discrete-time dynamics of the system assigned to vertex $\varsigma_{i} \in \mathcal{S}$ are governed by the difference equation

$$
x_{i}(k+1)=\phi_{i}\left(x_{i}(k), u_{i}(k), v_{i}\left(x_{\mathcal{N}_{i}}(k)\right)\right), \quad k \in \mathbb{Z}_{+},
$$

for vertex indices $i \in \mathcal{I}:=\mathbb{Z}_{[1, N]}$. Here, $x_{i} \in \mathbb{X}_{i} \subseteq \mathbb{R}^{n_{i}}$ is the state and $u_{i} \in \mathbb{U}_{i} \subseteq \mathbb{R}^{m_{i}}$ is the control input of the $i$-th system, i.e., the system assigned to vertex $\varsigma_{i}$. With each edge $\left(\varsigma_{j}, \varsigma_{i}\right) \in \mathcal{E}$ we associate a function $v_{i j}: \mathbb{R}^{n_{j}} \rightarrow \mathbb{R}^{n_{v_{i j}}}$ that defines the interconnection $v_{i j}\left(x_{j}(k)\right) \in \mathbb{R}^{n_{v_{i j}}}, k \in \mathbb{Z}_{+}$, between systems $j$ and $i$, i.e., $v_{i j}\left(x_{j}(k)\right)$ characterizes how the states of system $j$ influence the dynamics of system $i$. We use $\mathcal{N}_{i}:=\left\{j \mid\left(\varsigma_{j}, \varsigma_{i}\right) \in \mathcal{E}\right\}$ to denote the set of indices of the direct neighbors of system $i$. A direct neighbor of system $i$ is any system in the network whose dynamics (e.g., states or outputs) appear explicitly, via $v_{i j}(\cdot)$, in the state equations that govern the dynamics of system $i$. Let $\overline{\mathcal{N}}_{i}:=\mathcal{N}_{i} \cup\{i\}$. We define $x_{\mathcal{N}_{i}}(k):=\operatorname{col}\left(\left\{x_{j}(k)\right\}_{j \in \mathcal{N}_{i}}\right)$ as the vector that collects all the state vectors of the direct neighbors of system $i$ and $v_{i}\left(x_{\mathcal{N}_{i}}(k)\right):=\operatorname{col}\left(\left\{v_{i j}\left(x_{j}(k)\right)\right\}_{j \in \mathcal{N}_{i}}\right) \in \mathbb{R}^{n_{v_{i}}}$ as the vector that collects all the vector-valued interconnection signals that enter system $i$. The functions $\phi_{i}(\cdot, \cdot, \cdot)$ and $v_{i j}(\cdot)$ are nonlinear and satisfy $\phi_{i}\left(\mathbf{0}_{n_{i}}, \mathbf{0}_{m_{i}}, \mathbf{0}_{n_{v_{i}}}\right)=\mathbf{0}_{n_{i}}$ for all $i \in \mathcal{I}$ and $v_{i j}\left(\mathbf{0}_{n_{j}}\right)=\mathbf{0}_{n_{v_{i j}}}$ for all $(i, j) \in \mathcal{I} \times \mathcal{N}_{i}$. For all $i \in \mathcal{I}$ we assume that $\mathbf{0}_{n_{i}} \in \operatorname{int}\left(\mathbb{X}_{i}\right)$ and $\mathbf{0}_{m_{i}} \in \operatorname{int}\left(\mathbb{U}_{i}\right)$.

Finally, let the dynamics of the overall network of interconnected systems (4) be written in a compact form as

$$
x(k+1)=\phi(x(k), u(k)), \quad k \in \mathbb{Z}_{+}
$$

where $x=\operatorname{col}\left(\left\{x_{i}\right\}_{i \in \mathcal{I}}\right) \in \mathbb{R}^{n}, n=\sum_{i \in \mathcal{I}} n_{i}$, and $u=$ $\operatorname{col}\left(\left\{u_{i}\right\}_{i \in \mathcal{I}}\right) \in \mathbb{R}^{m}, m=\sum_{i \in \mathcal{I}} m_{i}$.

\section{A. Structured max-CLFS}

Next, consider the following definition, see also [15], [18].

Definition III.1 Let $\alpha_{1}^{i}, \alpha_{2}^{i} \in \mathcal{K}_{\infty}$ for $i \in \mathcal{I}$ and let $\left\{V_{i}\right\}_{i \in \mathcal{I}}$ be a set of functions $V_{i}: \mathbb{R}^{n_{i}} \rightarrow \mathbb{R}_{+}$that satisfy

$$
\alpha_{1}^{i}\left(\left\|x_{i}\right\|\right) \leq V_{i}\left(x_{i}\right) \leq \alpha_{2}^{i}\left(\left\|x_{i}\right\|\right)
$$

for all $x_{i} \in \mathbb{R}^{n_{i}}, i \in \mathcal{I}$. Then, given $\rho_{i} \in \mathbb{R}_{[0,1)}, i \in \mathcal{I}$, if there exists a set of control laws $\pi_{i}: \mathbb{R}^{n_{i}} \times \mathbb{R}^{n_{v_{i}}} \rightrightarrows \mathbb{U}_{i}$ such that

$$
V_{i}\left(\phi_{i}\left(x_{i}, u_{i}, v_{i}\left(x_{\mathcal{N}_{i}}\right)\right)\right) \leq \rho_{i} \max _{j \in \overline{\mathcal{N}}_{i}} V_{j}\left(x_{j}\right),
$$

for all $x_{i} \in \mathbb{X}_{i}, u_{i} \in \pi_{i}\left(x_{i}, v_{i}\left(x_{\mathcal{N}_{i}}\right)\right)$, the set $\left\{V_{i}\right\}_{i \in \mathcal{I}}$ is called a set of "structured max control Lyapunov functions" in $\mathbb{X}:=\left\{\operatorname{col}\left(\left\{x_{i}\right\}_{i \in \mathcal{I}}\right) \mid x_{i} \in \mathbb{X}_{i}\right\}$ for system (5).
In Def. III.1, the term structured emphasizes that each $V_{i}$ is a function of $x_{i}$ only, i.e., the set $\left\{V_{i}\right\}_{i \in \mathcal{I}}$ reflects the structural decomposition of the dynamics of overall interconnected system (4). Moreover, the term max originates from the corresponding convergence condition, i.e., (6b). Next, based on Def. III.1, we formulate the following feasibility problem.

Problem III.2 Let $\rho_{i} \in \mathbb{R}_{[0,1)}, i \in \mathcal{I}$, and a set of candidate structured max-CLFs $\left\{V_{i}\right\}_{i \in \mathcal{I}}$ be given. At time $k \in \mathbb{Z}_{+}$, let $\left\{x_{i}(k)\right\}_{i \in \mathcal{I}},\left\{v_{i}\left(x_{\mathcal{N}_{i}}(k)\right)\right\}_{i \in \mathcal{I}}$ and $\left\{V_{i}\left(x_{i}(k)\right)\right\}_{i \in \mathcal{I}}$ be known, and calculate a set of control actions $\left\{u_{i}(k)\right\}_{i \in \mathcal{I}}$, such that

$$
\begin{array}{r}
u_{i}(k) \in \mathbb{U}_{i}, \phi_{i}\left(x_{i}(k), u_{i}(k), v_{i}\left(x_{\mathcal{N}_{i}}(k)\right)\right) \in \mathbb{X}_{i}, \\
\left.V_{i}\left(\phi_{i}\left(x_{i}(k), u_{i}(k), v_{i}\left(x_{\mathcal{N}_{i}}(k)\right)\right)\right)\right) \\
\leq \rho_{i} \max _{j \in \overline{\mathcal{N}}_{i}} V_{j}\left(x_{j}(k)\right),
\end{array}
$$

for all $i \in \mathcal{I}$.

It can be proven that a feedback law $\pi: \mathbb{R}^{n} \rightarrow \mathbb{R}^{m}$ that selects an arbitrary control action $\operatorname{col}\left(\left\{u_{i}\right\}_{i \in \mathcal{I}}\right)$ out of the set of solutions to Prob. III.2 for each $x \in \mathbb{R}^{n}$ asymptotically stabilizes the difference equation $x(k+1)=$ $\phi(x(k), \pi(x(k)))$ in $\mathbb{X}$. This proof, given in [15], exploits the fact that the function $V(x):=\max _{i \in \mathcal{I}} V_{i}\left(x_{i}\right)$ is a CLF for the overall network if (7) is recursive feasible. The result then directly follows from Thm. I.3, given in the Appendix.

Note that in Prob. III.2, the functions $V_{i}$ do not need to be CLFs in $\mathbb{X}_{i}$ (conform Def. I.4) for each respective system $i \in \mathcal{I}$. Indeed, (7b) allows $V_{i}$ to increase, as long as for each system $i$ the value of $V_{i}$ at the next time instant is less than $\rho_{i}$ times the maximum over the current values of its own function and those of its direct neighbors. Still, (7b) may be restrictive in practice, as it can be hard to find $\left\{V_{i}\right\}_{i \in \mathcal{I}}$ that satisfy (6) for all $x_{i} \in \mathbb{X}_{i}$. Hence, we formulate the following feasibility problem, which permits non-monotonic decrease of both local functions $V_{i}\left(x_{i}\right)$ and $V(x)$.

Problem III.3 Given $N_{\tau} \in \mathbb{Z}_{\geq 1}$, consider Prob. III.2 for a set of structured max-CLFs $\left\{\bar{V}_{i}\right\}_{i \in \mathcal{I}}$ in $\widetilde{\mathbb{X}} \subset \mathbb{X}$, with (7b) replaced by

$$
\begin{aligned}
& V_{i}\left(\phi_{i}\left(x_{i}(k), u_{i}(k), v_{i}\left(x_{\mathcal{N}_{i}}(k)\right)\right)\right) \\
& \quad \leq \rho_{i} \max _{\tau \in \mathbb{Z}_{\left[0, N_{\tau}-1\right]}} \max _{j \in \overline{\mathcal{N}}_{i}} V_{j}\left(x_{j}(k-\tau)\right),
\end{aligned}
$$

for all $k \in \mathbb{Z}_{\geq N_{\tau}-1}$ and $i \in \mathcal{I}$.

In [15] it is proven that a feedback law $\bar{\pi}: \mathbb{R}^{n} \rightarrow \mathbb{R}^{m}$ that selects an arbitrary control action $\operatorname{col}\left(\left\{u_{i}\right\}_{i \in \mathcal{I}}\right)$ out of the set of solutions to Prob. III.3 for each $x \in \mathbb{R}^{n}$ renders the closed-loop system $x(k+1)=\phi(x(k), \bar{\pi}(x(k)))$ asymptotically stable in $\mathbb{X}$. The proof demonstrates that the function $V(x):=\max _{i \in \mathcal{I}} V_{i}\left(x_{i}\right)$ asymptotically converges to 0 for $k \rightarrow \infty$, assuming recursively feasibility of (7a) and (8). This and (6a) imply attractivity and Lyapunov stability.

Next, note that Prob. III.2 and Prob. III.3 are separable in $\left\{u_{i}\right\}_{i \in \mathcal{I}}$. Therefore, it is possible to compute the control action $u(k):=\operatorname{col}\left(\left\{u_{i}(k)\right\}_{i \in \mathcal{I}}\right)$ by solving $N$ feasibility problems independently, with each subproblem in $u_{i}(k)$ assigned to one local controller, corresponding to one system 
$i \in \mathcal{I}$. In order to compute $u_{i}(k)$, each controller needs to measure/estimate the current local state $x_{i}(k)$ and have knowledge of the interconnection terms $v_{i j}\left(x_{j}(k)\right), j \in$ $\mathcal{N}_{i}$, and the values $V_{j}\left(x_{j}(k)\right), j \in \overline{\mathcal{N}}_{i}$. In practice, many interconnection signals can be measured directly at node $i$, whereas a single run of information exchange among direct neighbors per sampling instant is sufficient to acquire the non-locally measurable signals. Notice that this is certainly the case in electrical power systems, where each generator bus represents a dynamical system, and the interconnection term may be the frequency of adjacent generator buses and the power flowing from/to these neighbors. The power flow $P_{\text {tie }_{i j}}(k)$ is directly measurable at node $i$, whereas the frequency $\omega_{j}(k)$ can only be determined at the corresponding bus and needs to be transmitted to node $i$. The values $V_{j}\left(x_{j}\right)$, $j \in \mathcal{N}_{i}$, can be computed both at node $j$ and $i$, although the latter option requires $j$ to send its full state information $x_{j}$ to $i$, instead of only $V_{j}\left(x_{j}\right)$. Note that the above described exchange of information between possibly different market actors does not carry competitive risks, as specific system parameters cannot be deduced from state information and $V_{j}\left(x_{j}\right)$ alone. This makes Prob. III.2 and Prob. III.3 well suited for use in a liberalized market environment.

If we combine Prob. III.3 with the optimization of a set of local cost functions, the feasibility-based stability guarantee and the possibility of an almost-decentralized implementation still hold. This enables the formulation of a one-stepahead predictive control algorithm in which stabilization is decoupled from performance, and in which the controllers do not need to attain the global optimum at each sampling instant, as typically required for stability in classical MPC. Given a set of convex objective functions $\left\{J_{i}\left(x_{i}, u_{i}\right)\right\}_{i \in \mathcal{I}}$, we thus consider the following algorithm.

Algorithm III.4 At each instant $k \in \mathbb{Z}_{+}$and node $i \in \mathcal{I}$ :

Step 1: Measure or estimate the current local state $x_{i}(k)$ and transmit $v_{j i}\left(x_{i}(k)\right)$ and $V_{i}\left(x_{i}(k)\right)$ to nodes $\left\{j \in \mathcal{I} \mid i \in \mathcal{N}_{j}\right\}$. Step 2: Minimize $J_{i}\left(x_{i}(k), u_{i}(k)\right)$ over the set of feasible local control actions (determined by Prob. III.3) and denote the optimizer by $u_{i}^{*}(k)$;

Step 3: Use $u_{i}(k)=u_{i}^{*}(k)$ as control action.

The interested reader is referred to [15] for more information on the algorithms and results presented in this section.

\section{B. Implementation Issues}

For infinity-norm based CLFs (i.e., $V_{i}\left(x_{i}\right)=\left\|P_{i} x_{i}\right\|_{\infty}$, with full-column rank $P_{i} \in \mathbb{R}^{p_{i} \times n_{i}}$ ) and input-affine prediction models $x_{i}(k+1)=f_{i}\left(x_{i}(k), v_{i}\left(x_{\mathcal{N}_{i}}(k)\right)\right)+$ $g_{i}\left(x_{i}(k), v_{i}\left(x_{\mathcal{N}_{i}}(k)\right)\right) u_{i}(k),(8)$ can be formulated as a set of linear inequalities without introducing conservatism. By definition of the infinity norm, for $\|x\|_{\infty} \leq c$ to hold for some $x \in \mathbb{R}^{n}$ and $c \in \mathbb{R}$, it is necessary and sufficient to require that $\pm[x]_{j} \leq c$ for all $j \in \mathbb{Z}_{[1, n]}$. Hence, (8) is satisfied if and only if, for all $j \in \mathbb{Z}_{\left[1, p_{i}\right]}$ and $k \in \mathbb{Z}_{\geq N_{\tau}-1}$,

$$
\begin{aligned}
& \pm\left[P_{i}\left\{g_{i}\left(x_{i}(k), v_{i}\left(x_{\mathcal{N}_{i}}(k)\right)\right) u_{i}(k)\right\}\right]_{j} \\
& \quad \leq \zeta_{i}(k) \mp\left[P_{i}\left\{f_{i}\left(x_{i}(k), v_{i}\left(x_{\mathcal{N}_{i}}(k)\right)\right)\right\}\right]_{j},
\end{aligned}
$$

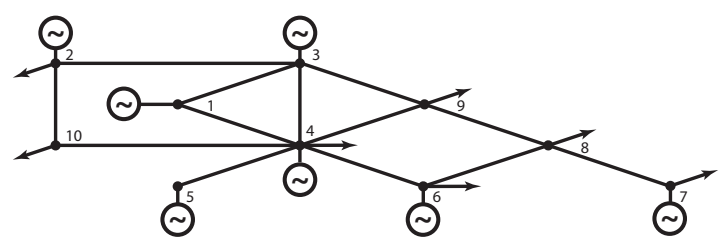

Fig. 1. Single-line representation of the seven-machine CIGRÉ test system.

TABLE I

\begin{tabular}{|c|c|}
\hline$H_{1}, \ldots, H_{7}$ & $100,30.3,35.8,28.6,26,34.8,26.4$ \\
\hline$D_{1}, \ldots, D_{7}$ & $0.8,0.85,0.8,0.8,0.9,0.7,0.8$ \\
\hline$\tau_{\mathrm{G}_{1}}, \ldots, \tau_{\mathrm{G}_{7}}$ & $0.2,0.15,0.2,0.2,0.25,0.2,0.2$ \\
\hline$\tau_{\mathrm{T}_{1}}, \ldots, \tau_{\mathrm{T}_{7}}$ & $0.5,0.4,0.5,0.5,0.4,0.5,0.5$ \\
\hline$b_{i j}$ & $\begin{array}{l}b_{1,3}=24.5, b_{1,4}=24.5, b_{2,3}=62.6, b_{2,10}=32.3 \\
b_{3,4}=40, b_{3,9}=28, b_{4,5}=10, b_{4,6}=10, b_{4,9}=97 \\
b_{4,10}=33, b_{6,8}=31.8, b_{7,8}=39.5, b_{8,9}=97\end{array}$ \\
\hline$r_{1}, \ldots, r_{7}$ & $\frac{1}{20}, \frac{1}{23}, \frac{1}{19}, \frac{1}{21}, \frac{1}{21}, \frac{1}{18}, \frac{1}{20}$ \\
\hline $\mathcal{N}_{1}, \ldots, \mathcal{N}_{7}$ & $\begin{array}{l}\{3,4\},\{3,4\},\{1,2,4,6,7\},\{1,2,3,5,6,7\}, \\
\{4\},\{3,4,7\},\{3,4,6\}\end{array}$ \\
\hline $\begin{array}{l}F_{1}, \ldots, F_{7} \\
\quad \times 10\end{array}$ & 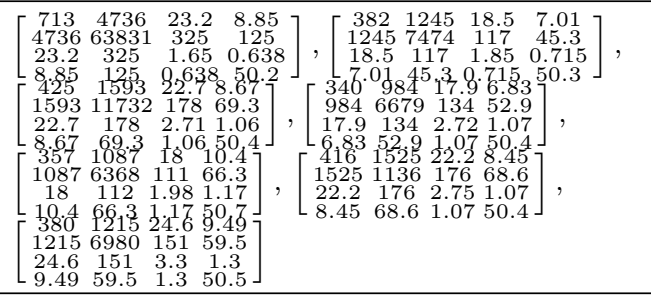 \\
\hline$Q_{1}, \ldots, Q_{7}$ & $Q_{i}=100 \cdot \operatorname{diag}(5,5,0,0), i \in \mathcal{I}$ \\
\hline$R_{1}, \ldots, R_{7}$ & $R_{i}=0.1, i \in \mathcal{I}$ \\
\hline
\end{tabular}

SIMULATION PARAMETERS

where $\zeta_{i}(k):=\rho_{i} \max _{\tau \in \mathbb{Z}_{\left[0, N_{\tau}-1\right]}} \max _{j \in \overline{\mathcal{N}}_{i}} V_{j}\left(x_{j}(k-\tau)\right) \in$ $\mathbb{R}_{+}$is constant for any $k \in \mathbb{Z}_{\geq N_{\tau}-1}$. This yields a total of $2 p_{i}$ linear inequalities in $u_{i}$. In combination with polytopic state/input sets and an infinity-norm or quadratic cost function, it is therefore possible to implement step 2 of Alg. III.4 as a linear or quadratic program, respectively.

\section{Application CASE Study}

We illustrate the potential of Alg. III.4 for application in frequency control by simulating it in closed-loop with the 7-machine CIGRÉ (International Council on Large Electric Systems) test power system reproduced from [19]. The network is schematically depicted in Fig. 1, and consists of $N=7$ generator buses and 3 load buses, interconnected via 13 transmission lines. Each generator is modeled in accordance with (1a), (1c)-(1d) and (3). The parameters used in the simulation are listed in Table I.

The prediction model employed by Alg. III.4, i.e., (4), is obtained via time discretization of (1a), (1c)-(1d) and (3), using sampling period $T_{\mathrm{s}}=1 \mathrm{~s}$. This yields the discrete-time linear state-space representation

$$
\begin{aligned}
x_{i}(k+1) & =\phi_{i}\left(x_{i}(k), u_{i}(k), v_{i}\left(x_{\mathcal{N}_{i}}(k)\right)\right) \\
& :=A_{i i} x_{i}(k)+B_{i i} u_{i}(k)+v_{i}\left(x_{\mathcal{N}_{i}}(k)\right), \\
v_{i}\left(x_{\mathcal{N}_{i}}(k)\right) & :=\sum_{j \in \mathcal{N}_{i}} A_{i j} x_{j}(k),
\end{aligned}
$$


for $x_{i}:=\left[\begin{array}{llll}\delta_{i} & \omega_{i} & P_{\mathrm{M}_{i}} & P_{\mathrm{G}_{i}}\end{array}\right]^{\top}, u_{i}:=P_{\mathrm{ref}_{i}}, i \in \mathcal{I}:=\{1, \ldots, 7\}$, and where $A_{i i} \in \mathbb{R}^{n_{i} \times n_{i}}, B_{i i} \in \mathbb{R}^{n_{i} \times m_{i}}, A_{i j} \in \mathbb{R}^{n_{i} \times n_{j}}$.

In order to compute $x_{i}(k+1)$, controller $i$ needs to have knowledge of $v_{i j}\left(x_{j}(k)\right)$ and $V_{j}\left(x_{j}(k)\right), j \in \mathcal{N}_{i}$, where the set of direct neighbors $\mathcal{N}_{i}$ is given in Table I. The direct neighbors of generator $i$ are those generator buses $j$ that are connected by a single edge $\varepsilon_{i j} \in \overline{\mathcal{E}}$, or, due to loadbus elimination in single-area control problems, by a path in single-line diagram $\overline{\mathcal{G}}$ from generator $i$ to generator $j$ via load buses only. Consequently, some of the sparsity of non-reduced network graph $\overline{\mathcal{G}}$ is lost. Note that load-bus elimination is not required if multiple generator and load buses are clustered into control areas that are governed by difference equations only (i.e., with no need for algebraic relations). This is the case, e.g., in Europe, where the multi-area secondary control problem is solved by assigning a separate controller to every country/area, each usually connected to a small number of neighboring areas only.

The controllers employ quadratic cost functions, i.e., $J_{i}\left(x_{i}, u_{i}\right):=\left(x_{i}^{+}\right)^{\top} F_{i} x_{i}^{+}+x_{i}^{\top} Q_{i} x_{i}+u_{i}^{\top} R_{i} u_{i}, i \in \mathcal{I}$, with one-step-ahead state prediction $x_{i}^{+}:=\phi_{i}\left(x_{i}, u_{i}, v_{i}\left(x_{\mathcal{N}_{i}}\right)\right)$. Table I lists the values of $F_{i} \succ 0, Q_{i} \succeq 0$ and $R_{i} \succ$ 0 . Note that $F_{i}$ satisfies the discrete-time Riccati equation $F_{i}=A_{i i}^{\top} F_{i} A_{i i}+A_{i i}^{\top} F_{i} B_{i i} L_{i}+Q_{i}$, with linear quadratic regulator feedback gain $L_{i}=\left(R_{i}+B_{i i}^{\top} F_{i} B_{i i}\right)^{-1} B_{i i}^{\top} F_{i} A_{i i}$. This specific value was chosen to optimize performance in terms of $\sum_{k \in \mathbb{Z}_{+}} \sum_{i \in \mathcal{I}} x_{i}(k)^{\top} Q_{i} x_{i}(k)+u_{i}(k) R_{i} u_{i}(k)$, where $Q_{i}$ penalizes $\delta_{i}$ and $\omega_{i}$ to induce adequate frequency and line flow damping. Yet, in contrast to standard MPC, $F_{i}$ 's value is irrelevant for guaranteeing closed-loop stability.

The method of [20] was used to compute the weights $P_{i} \in$ $\mathbb{R}^{n_{i} \times n_{i}}, i \in \mathcal{I}$, of the local infinity-norm based candidate CLFs for Alg. III.4, i.e., $V_{i}\left(x_{i}\right)=\left\|P_{i} x_{i}\right\|_{\infty}$ with $\rho_{i}=0.9$, $\forall i \in \mathcal{I}$, and system (10), in closed-loop with local feedback laws $u_{i}(k):=K_{i} x_{i}(k), K_{i} \in \mathbb{R}^{1 \times n_{i}}$, yielding

$$
\begin{array}{rlrl}
P_{1}= & {\left[\begin{array}{cccc}
-0.62979 & 17.158 & 7.3639 & -6.5897 \\
-1.2466 & 38.485 & 0.68023 & 2.3994 \\
3.0898 & 33.119 & 0.35449 & 0.94405 \\
-0.41334 & -14.006 & 0.11274 & 0.99418
\end{array}\right]} & K_{1}=\left[\begin{array}{c}
-0.056146 \\
17.013 \\
0.17313 \\
0.63512
\end{array}\right]^{\top} \\
P_{2}= & {\left[\begin{array}{cccc}
-7.7327 & 24.86 & -0.45184 & -1.3596 \\
9.6994 & 62.824 & 10.53 & -11.793 \\
9.7231 & 72.123 & -2.791 & 9.8619 \\
-8.0183 & 4.4586 & 7.5088 & 1.19
\end{array}\right]} & K_{2}=\left[\begin{array}{c}
-0.1415 \\
17.27 \\
-0.42816 \\
1.1958
\end{array}\right]^{\top} \\
P_{3}= & {\left[\begin{array}{cccc}
-0.94289 & 34.854 & -6.4203 & 10.729 \\
-5.7409 & -16.222 & 1.6906 & -0.96636 \\
6.8157 & 44.128 & 4.5873 & -3.129 \\
12.125 & 10.053 & 0.8673 & 0.68066
\end{array}\right] K_{3}=\left[\begin{array}{c}
-1.9488 \\
10.154 \\
0.15808 \\
0.20908
\end{array}\right]^{\top}} \\
P_{4}= & {\left[\begin{array}{cccc}
1.6663 & 7.5646 & -5.1264 & 13.222 \\
4.0893 & -20.416 & -0.89703 & -0.58409 \\
-1.9142 & 2.2781 & 7.2915 & -1.6996 \\
4.0666 & 29.068 & 0.84367 & 0.18502
\end{array}\right] K_{4}=\left[\begin{array}{c}
0.11742 \\
17.745 \\
0.37284 \\
0.12559
\end{array}\right]^{\top}} \\
P_{5}= & {\left[\begin{array}{cccc}
5.7046 & 5.5612 & -5.5749 & 7.2372 \\
6.5151 & 25.236 & 1.0041 & -0.082996 \\
-5.2836 & 18.191 & 1.1193 \\
4.8618 & -12.936 & 0.42708 & 0.18971 \\
-0.1392
\end{array}\right] K_{5}=\left[\begin{array}{c}
-0.13866 \\
14.185 \\
0.5927 \\
-0.20511
\end{array}\right]^{\top}} \\
P_{6}= & {\left[\begin{array}{cccc}
-2.7264 & 10.554 & 1.1353 & 3.9908 \\
2.5412 & -12.799 & 0.68144 & -0.30603 \\
2.7504 & 4.5373 & -4.8496 & 3.1844 \\
3.4173 & 21.908 & 0.28121 & 0.062939
\end{array}\right]} & K_{6}=\left[\begin{array}{c}
-0.17089 \\
11.615 \\
0.072399 \\
0.038927
\end{array}\right]^{\top} \\
P_{7}= & {\left[\begin{array}{cccc}
5.6752 & 54.244 & 4.6571 & -0.39439 \\
-0.26188 & 23.17 & -3.3943 & 13.382 \\
8.5394 & 28.707 & 2.5505 & 4.1042 \\
0.044767 & 3.4706 & 9.2078 & -2.4838
\end{array}\right]} & K_{7}=\left[\begin{array}{c}
-0.4908 \\
15.387 \\
0.62681 \\
-0.229
\end{array}\right]^{\top} .
\end{array}
$$

It is important to stress that the control laws $u_{i}(k)=$ $K_{i} x_{i}(k)$ are only employed off-line, to calculate the matrices $P_{i}$ and they are never used for controlling the system. Moreover, we set $N_{\tau}=5$ in Alg. III.4. Note that by choosing infinity-norm CLFs, it is possible to formulate Alg. III.4 as
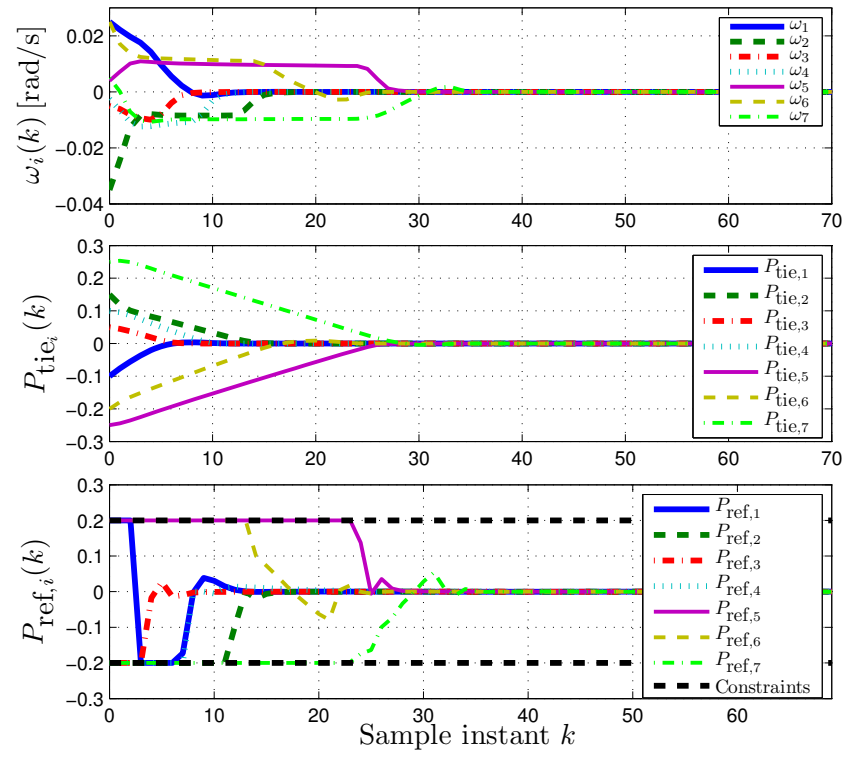

Fig. 2. Frequency, flows and inputs under structured max-CLF control.

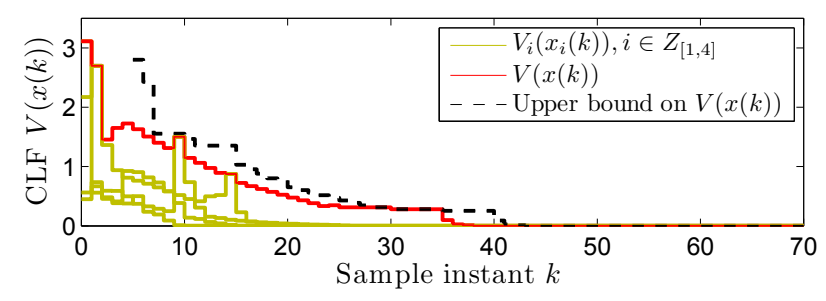

Fig. 3. Evolution of $V_{i}\left(x_{i}(k)\right), V(x(k))$ and its upper bound over time.

a quadratic program (QP), as explained in Section III-B.

In the simulation we evaluated the performance of the closed-loop network when recovering from a state perturbation (or imbalance) given by

$\left\{x_{i}(0)\right\}_{i \in \mathcal{I}}=10^{-2} \times$

$\left\{\left[\begin{array}{c}-10 \\ 2.5 \\ -40 \\ 10\end{array}\right],\left[\begin{array}{c}15 \\ -3.5 \\ 1.5 \\ 10\end{array}\right],\left[\begin{array}{c}5 \\ -0.5 \\ 0 \\ 1\end{array}\right],\left[\begin{array}{c}10 \\ -0.25 \\ 1 \\ 0.05\end{array}\right],\left[\begin{array}{c}-25 \\ 0.4 \\ 0 \\ 5\end{array}\right],\left[\begin{array}{c}-20 \\ 2 \\ -50 \\ 0.1\end{array}\right],\left[\begin{array}{c}25 \\ 0.5 \\ 1.5 \\ -4.5\end{array}\right]\right\}$.

Furthermore, we assume a nominal, static load, i.e., we set $P_{\mathrm{L}_{i}}(k):=0$ for $k \in \mathbb{Z}_{+}$and $i \in \mathcal{I}$. Since power networks are generally subject to constraints, for physical, performance or safety reasons, we constrain the control inputs as

$$
-0.2 \leq u_{i} \leq 0.2, i \in \mathcal{I}
$$

The relevant system outputs $\left(\omega_{i}, P_{\text {tie }_{i}}\right)$, where $P_{\text {tie }_{i}}(t):=$ $\sum_{\left\{j \mid\left(\varsigma_{i}, \varsigma_{j}\right) \in \mathcal{E}\right\}} P_{\mathrm{tie}_{i j}}(t)$, are shown in Fig. 2, along with the corresponding control inputs $P_{\text {ref }_{i}}, i \in \mathcal{I}$. Clearly, the trajectories converge to 0 for $k \rightarrow \infty$. Note that constraint (11) is not violated, although it is active for some time instants. Fig. 3 depicts the evolution of $V_{i}\left(x_{i}(k)\right)$ for $i \in \mathbb{Z}_{[1,4]}$, $V(x(k))$ and the corresponding upper bound generated by condition (8) in Alg. III.4. The simulation shows that $V(x(k))$ may vary arbitrarily within the converging envelope defined by (8), resulting in closed-loop stability. 


\section{CONCLUSIONS}

Stable operation of future electrical power systems will require advanced control techniques for supply/demand balancing, as a consequence of the liberalization and decentralization of electrical power generation. In this paper we studied an almost-decentralized Lyapunov-based predictive control algorithm for load-frequency control, i.e., for asymptotic grid-frequency stabilization. The scheme is particularly suited for large-scale power networks, as it only requires local information and short-distance communication between directly-coupled generator buses to provide a stabilizing control action. We assessed the potential of the almostdecentralized predictive control scheme for practical application by simulating it in closed-loop with the 7-machine CIGRÉ benchmark system. The obtained simulation results encourage further development of the almost-decentralized MPC method for application in power system control, as its performance matches that of conventional frequency-control schemes while offering a closed-loop stability guarantee.

\section{APPENDIX I \\ LYAPUNOV STABILITY}

Consider the discrete-time autonomous system

$$
x(k+1)=\Phi(x(k)), k \in \mathbb{Z}_{+},
$$

where $x(k) \in \mathbb{X} \subseteq \mathbb{R}^{n}$ is the state at discrete-time instant $k \in \mathbb{Z}_{+}$. The nonlinear function $\Phi: \mathbb{R}^{n} \rightarrow \mathbb{R}^{n}$ satisfies $\Phi\left(\mathbf{0}_{n}\right)=\mathbf{0}_{n}$, i.e., the origin is an equilibrium of (12).

Definition I.1 A set $\mathcal{P} \subseteq \mathbb{R}^{n}$ is Positively Invariant (PI) for system (12) if $\forall x \in \mathcal{P}$ it holds that $\Phi(x) \in \mathcal{P}$.

Definition I.2 (i) System (12) is Lyapunov stable if $\forall \varepsilon>0$, $\exists \delta(\varepsilon)>0$ such that for all trajectories of (12) it holds that $\|x(0)\| \leq \delta(\varepsilon) \Rightarrow\|x(k)\| \leq \varepsilon$ for all $k \in \mathbb{Z}_{+}$. (ii) Let $\mathbb{X} \subseteq \mathbb{R}^{n}$ and $\mathbf{0}_{n} \in \operatorname{int}(\mathbb{X})$. The origin is attractive in $\mathbb{X}$ if for any $x(0) \in \mathbb{X}$ it holds that all corresponding trajectories of (12) satisfy $\lim _{k \rightarrow \infty}\|x(k)\|=0$. (iii) System (12) is asymptotically stable in $\mathbb{X}(\operatorname{AS}(\mathbb{X}))$ if it is Lyapunov stable and attractive in $\mathbb{X}$.

Theorem I.3 Let $\mathbb{X}$ be a PI set for system (12) and let $\mathbf{0}_{n} \in$ $\operatorname{int}(\mathbb{X})$. Furthermore, let $\alpha_{1}, \alpha_{2} \in \mathcal{K}_{\infty}, \rho \in \mathbb{R}_{[0,1)}$ and let $V$ : $\mathbb{R}^{n} \rightarrow \mathbb{R}_{+}$be a function such that

$$
\begin{aligned}
\alpha_{1}(\|x\|) \leq V(x) & \leq \alpha_{2}(\|x\|) \\
V\left(x^{+}\right) & \leq \rho V(x)
\end{aligned}
$$

for all $x \in \mathbb{X}$ and all $x^{+}=\Phi(x)$. Then (12) is AS(X).

A function $V$ that satisfies the conditions of Thm. I.3 is called a Lyapunov function.

\section{A. CLFs for discrete-time systems}

Consider the discrete-time constrained nonlinear system

$$
x(k+1)=\phi(x(k), u(k)), \quad k \in \mathbb{Z}_{+},
$$

where $x(k) \in \mathbb{X} \subseteq \mathbb{R}^{n}$ is the state and $u(k) \in \mathbb{U} \subseteq \mathbb{R}^{m}$ is the control input at discrete-time instant $k \in \mathbb{Z}_{+}$. The function $\phi: \mathbb{R}^{n} \times \mathbb{R}^{m} \rightarrow \mathbb{R}^{n}$ is nonlinear with $\phi\left(\mathbf{0}_{n}, \mathbf{0}_{m}\right)=\mathbf{0}_{n}$. We assume that $\mathbb{X}$ and $\mathbb{U}$ are bounded sets with $\mathbf{0}_{n} \in \operatorname{int}(\mathbb{X})$ and $\mathbf{0}_{m} \in \operatorname{int}(\mathbb{U})$. Next, let $\alpha_{1}, \alpha_{2} \in \mathcal{K}_{\infty}$ and $\rho \in \mathbb{R}_{[0,1)}$.

Definition I.4 A function $V: \mathbb{R}^{n} \rightarrow \mathbb{R}_{+}$that satisfies $\alpha_{1}(\|x\|) \leq V(x) \leq \alpha_{2}(\|x\|), \forall x \in \mathbb{R}^{n}$, for which there exists a control law $\pi: \mathbb{R}^{n} \rightrightarrows \mathbb{U}$ such that $V(\phi(x, u)) \leq$ $\rho V(x), \forall x \in \mathbb{X}, \forall u \in \pi(x)$, is a control Lyapunov function (CLF) in $\mathbb{X}$ for (14).

\section{ACKNOWLEDGEMENT}

This research is supported by SenterNovem/Agentschap NL and the Veni grant "Flexible Lyapunov Functions for Real-time Control", grant no. 10230, awarded by STW and NWO.

\section{REFERENCES}

[1] S. Stoft, Power System Economics: Designing Markets for Electricity. Kluwer Academic Publishers, 2002.

[2] A.-M. Borbely and J. F. Kreider, Distributed generation: the power paradigm for the new millennium. CRC Press, 2001.

[3] P. Kundur, Power system stability and control. McGraw-Hill, 1994.

[4] UCTE ad-hoc group, "Frequency quality investigation report," UCTE, Tech. Rep., 2008.

[5] T. Weißbach and E. Wellfonder, "High frequency deviations within the European power system: Origins and proposals for improvement," in Power Systems Conf. Exp., Seattle, WA, March 2009, pp. 1-6.

[6] A. N. Venkat, I. A. Hiskens, J. B. Rawlings, and S. J. Wright, "Distributed MPC strategies with application to power system automatic generation control," IEEE Transactions on Control Systems Technology, vol. 16, no. 6, pp. 1192-1206, 2008.

[7] E. Camponogara, D. Jia, B. H. Krogh, and S. Talukdar, "Distributed model predictive control," IEEE Control Systems Magazine, vol. 22, no. 1, pp. 44-52, 2002.

[8] A. Jokić, M. Lazar, and P. P. J. Van den Bosch, "Price-based optimal control of power flow in electrical energy transmission networks," in Hybrid Systems: Computation and Control, ser. L. Notes in Computer Science, vol. 4416. Pisa, Italy: Springer, 2007, pp. 315-328.

[9] A. Alessio and A. Bemporad, "Decentralized model predictive control of constrained linear systems," European Control Conference, pp. 2813-2818, 2007.

[10] L. Magni and R. Scattolini, "Stabilizing decentralized model predictive control of nonlinear systems," Automatica, vol. 42, no. 7, pp. 12311236, 2006.

[11] D. M. Raimondo, L. Magni, and R. Scattolini, "Decentralized MPC of nonlinear systems: An input-to-state stability approach," Int. J. Robust and Nonlinear Control, vol. 17, no. 17, pp. 1651-1667, 2007.

[12] A. Richards and J. P. How, "Robust distributed model predictive control," Int. J. Control, vol. 80, no. 9, pp. 1517-1531, 2007.

[13] M. Lazar, W. P. M. H. Heemels, and A. Jokić, "Self-optimizing robust nonlinear model predictive control," in Nonlinear Model Predictive Control, ser. L. Notes in Control and Information Sciences, vol. 348. Springer, 2009, pp. 27-40.

[14] A. C. R. M. Damoiseaux, A. Jokić, M. Lazar, A. Alessio, P. P. J. Van den Bosch, I. A. Hiskens, and A. Bemporad, "Assessment of decentralized model predictive control techniques for power networks," Power Systems Computation Conference, 2008.

[15] R. M. Hermans, M. Lazar, and A. Jokić, "Almost decentralized Lyapunov-based nonlinear model predictive control," in American Control Conference, Baltimore, MD, USA, 2010.

[16] R. D. Christie, B. F. Wollenberg, and I. Wangensteen, "Transmission management in the deregulated environment," Proceedings of the IEEE, vol. 88, no. 2, pp. 170-195, 2000.

[17] A. Bemporad, "A predictive controller with artificial Lyapunov function for linear systems with input/state constraints," Automatica, vol. 34, no. 10, pp. 1255-1260, 1998.

[18] A. Jokić and M. Lazar, "On decentralized stabilization of discrete-time nonlinear systems," in American Control Conference, St. Louis, USA, 2009, pp. 5777-5782.

[19] M. A. Pai, Power System Stability by Lyapunov's Method. Amsterdam: North-Holland Publishing Company, 1981.

[20] M. Lazar, W. P. M. H. Heemels, S. Weiland, and A. Bemporad, "Stabilizing model predictive control of hybrid systems," IEEE Transactions on Automatic Control, vol. 51, no. 11, pp. 1813-1818, 2006. 